American Journal of Infectious Diseases 2 (2): 74-79, 2006

ISSN 1553-6203

(C) 2006 Science Publications

\title{
Cortisol Response Mediates HIV-1-Related Cognitive Deficits Among Injecting Drug Abusers
}

\author{
Raymond L. Ownby, Drenna Waldrop-Valverde, Adarsh M. Kumar, Alison Mack \\ J. Benny Fernandez, Louis González, Peggy González and Mahendra Kumar \\ Department of Psychiatry and Behavioral Sciences \\ University of Miami Miller School of Medicine, Miami, Florida, USA
}

\begin{abstract}
The cortisol response is an important measure of the endocrine activity to environmental challenges and has been related to cognitive function and mood. Previous studies have shown that the cortisol response to stress is dysregulated in persons with HIV-1 infection. Since cortisol is neurotoxic and its levels have been related to cognitive dysfunction in various disorders, it is possible that neuroendocrine dysregulation may also be related to cognitive dysfunction in individuals with HIV-1 infection. The purpose of this study was to test the hypothesis that the cortisol response to an alpha adrenergic challenge, cold pressor, is related to cognitive function in HIV-infected injecting drug abusers. We used growth curve modeling to study the relationship of cold pressor challenge stimulated cortisol response to scores on the modified HIV Dementia Scale (mHDS). To test this hypothesis, we assessed the effects of HIV-1 infection on the HDS score directly and indirectly via pattern of cortisol response. The analysis showed that HIV-1 infection was directly related to mHDS performance and that it also influenced scores on the mHDS by way of individuals' pattern of cortisol response. Cortisol response to $\alpha$-adrenergic challenge thus may mediate cognitive deficits in individuals with HIV-1 infection. These findings further emphasize the importance of understanding the role of stress in the cognitive problems associated with HIV-1 infection.
\end{abstract}

Key words: Cortisol, cognition disorders, HIV, IDUs, cold pressor challenge

\section{INTRODUCTION}

Hypothalamic-pituitary-adrenal (HPA) axis activity is dependent upon an individual's level of distress. Knowledge of one's seroconversion to HIV-1 infection in the early days before the availability of anti-retroviral intervention was considered to be a highly stressful life event, but despite the current wide availability of highly active antiretroviral therapy, abnormalities in HPA axis activity are still observed among HIV-1+ individuals. In an earlier report, we proposed that HIV-1 infection should now be considered a chronic stressor. Moreover, on the basis of various reports, we proposed that changes in HPA axis activity may be a consequence of molecular mimicry between the HIV-1 genome and key control elements of the HPA axis ${ }^{[1]}$. For instance, it has been reported that the corticotrophin releasing hormone $(\mathrm{CRH})$ response element binding protein (REB) binds to a specific region of the POMC promoter with sequence CTGTGCGCGCAG. This POMC promoter is $100 \%$ homologous to a region of the HIV genome located just before the gag gene and has a $>80 \%$ homology with the long terminal repeat (LTR) region $^{[2,3]}$. Interestingly, it also has been observed that the HIV-pregag sequence can bind in vitro with the POMC promoter sequence ${ }^{[4]}$.
Dysregulation of cortisol response has profound effects not only on behavioral, circulatory, endocrine/metabolic and immune functions but also on cognitive functions ${ }^{[5-7]}$. Several studies have reported the occurrence of endocrine dysfunctions in HIV-1 infection ${ }^{[8-12]}$. ACTH and cortisol are important markers of neuroendocrine responses to different physical and psychological stressors. These stressors result in heightened $\alpha$ - and/or $\beta$-adrenergic responses. Both of these heightened activities stimulate the HPA axis, resulting in an increase in central CRH activity which, in turn, stimulates ACTH secretion and is followed by an increase in cortisol levels produced by the adrenal cortex. Cortisol, in turn, inhibits endogenous ACTH and CRH secretion via a negative feedback loop so that the deleterious effects of the stressors are curtailed. This model provides the theoretical basis for studying the effects of stressors on the neuroendocrine system.

In addition, the attenuated autonomic response observed in HIV-1 infection ${ }^{[13]}$ may be another route that affects the HPA axis, since integration of the central autonomic system with the CNS is essential for the activation of the HPA axis ${ }^{[14]}$. However, the kinetics of the cortisol response, rather than absolute concentrations of cortisol, are considered to be more important in the neurohormonal response to stress and successful adaptation to stressors ${ }^{[11]}$. In order to

Corresponding Author: $\quad$ Dr. Mahendra Kumar, Department of Psychiatry and Behavioral Sciences, Suite 2026 Elliott Building, P.O. Box 016960 (M817), Miami, FL 33101, Tel: (305) 243-5034, Fax: (305) 243-5035 
investigate the cortisol response, even though there are a number of behavioral and laboratory paradigms, this investigation used the cold pressor challenge since possible cognitive complications occurring in HIV-1 infection may confound the results of behavioral challenges involving speech or arithmetic paradigms.

Literature on reactivity to different challenges (tasks) in cardiovascular research suggests that baroreceptors on arteries transmit impulses to the central nervous system at frequencies related to fluctuating arterial pressure. After processing the signals in the nervous system, efferent impulses are delivered along with the autonomic nerves (e.g. vagus and sympathetic) and are distributed in different organs including the heart and peripheral vasculature. Catecholamines released at the nerve terminals, in response to efferent impulses, influence the rate and vigor of cardiac contraction and adjust the resistance to blood flow through the vasculature. Thus, autonomic signals and neurohormones play an important role in the regulation of blood pressure and cardiac function. The status of these systems only becomes evident, however, in response to challenge and may not show its inadequacy under basal conditions. If the observed autonomic system dysfunction is a result of HIV-1 infection, it would indicate a defect in the neural circuitry and the response to challenge could be related to HIV-1 infection. In cold pressor challenge studies where a limb is immersed in an ice/water mixture, HPA axis activity can be investigated in terms of plasma cortisol levels and the response can be quantified using growth curve modeling.

Growth curve modeling ${ }^{[15]}$ is a powerful method for analyzing change in some parameter of interest over time. A variant of structural equation modeling (SEM), growth curve modeling allows explicit estimation of individual patterns of change over time as a basic level variable (analogous to the intercept in standard linear regression) and a shape variable that estimates the pattern of change over time (analogous to the slope in linear regression). This approach allows estimation of the effects of external covariates on both the level and shape variables (analogous to multivariate analysis of variance, or MANOVA), but a distinct advantage of growth curve modeling is that the effect of level and shape variables on other variables can be estimated (in traditional MANOVA, this is not possible). The model thus allows great flexibility in estimating patterns of change, how variables affect the change and the impact of change on other variables.

The purpose of this study was to use growth curve modeling to examine the covariates and the effects of cortisol response in individuals who are HIV-1+ IDUs. We specifically evaluated the hypothesis that cognitive function in these individuals is mediated at least in part by cortisol response.

\section{MATERIALS AND METHODS}

Sample: All participants were community residing and were recruited from the community and streets of South Florida. Participants were between 18 and 50 years of age in order to reduce the potential influence of increased age on cortisol response. Participants were excluded from the study if they had a history of diabetes, poorly controlled hypertension, head injury with loss of consciousness for more than 30 minutes, or a history of major psychiatric illness such as schizophrenia or bipolar disorder. Participants (total $\mathrm{n}=$ 293) for this study were in one of three groups: (1) HIV-1 positive individuals with a history of injecting drug use (IDU; $\mathrm{n}=101$ ), (2) HIV-1 negative individuals with a history of IDU $(n=130)$, or HIV-1 negative individuals with no history of IDU $(n=62)$. All participants gave informed consent prior to initiation of the study and all participants were financially compensated for their participation.

Drug use inclusion and exclusion criteria: HIV-1 positive and HIV-1 negative IDUs were required to have injected drugs, i.e., heroin or cocaine, at least six times in any one year period. Additionally, control participants could not have a history of dependence on any substance as determined by the Structured Clinical Interview for DSM-IV Axis I Disorders ${ }^{[16]}$. All participants were required to have abstained from drugs and alcohol for at least twelve hours prior to the study and this was verified by self-report and urine toxicology screen for drug use. If a participant was found to be intoxicated, he or she was rescheduled for the study at a later date.

HIV infection inclusion and exclusion criteria: Serostatus of HIV-1+ participants was verified by examination of laboratory reports. Additionally, PCR amplicor method (Roche Diagnostics; the Clinical Immunology Laboratory in the Department of Medicine, the University of Miami, School of Medicine) was used to quantify peripheral plasma viral load. HIV-1+ participants were free of any AIDSdefining symptoms at the time of the study. Verification of HIV seronegative status was not available as part of this study.

Measures: General cognitive performance was measured with the modified HIV Dementia Scale $(\mathrm{mHDS})^{[17]}$. The Modified HIV Dementia Scale omits the Attention category, making the modified scale more ideal for administration by the psychometrician. This scale is a screening measure consisting of 4 items measuring speed of information processing, short-term memory and visuoconstructive abilities. This measure is sensitive to the cognitive deficits associated with subcortical dysfunction commonly found in persons with HIV infection. 
Cold pressor challenge: The cold pressor challenge was performed between 8:00 a.m. and 11:00 a.m. On arrival, an indwelling venous catheter for drawing blood was placed in the antecubital vein of each participant. After twenty minutes of rest in a reclined position, a $9 \mathrm{ml}$ sample of blood was drawn in a tube containing EDTA to determine the resting, baseline level (T0) of cortisol. The cold pressor challenge was then administered. Participants placed their entire hand in an ice/water mixture (three parts ice and one part water) for two minutes. Following completion of the cold pressor challenge, four additional blood samples were collected at ten minutes (T1), fifteen minutes (T2), thirty minutes (T3) and fifty minutes (T4) after baseline. Samples of blood were immediately centrifuged at $4^{\circ} \mathrm{C}$ and the plasma obtained was aliquoted, stored at $-80^{\circ} \mathrm{C}$ until assayed.

Cortisol quantification: A solid-phase radioimmunoassay (RIA) was used to quantify plasma cortisol concentration using a commercially available kit (DSL, Webster, TX). Briefly, samples of plasma were defrosted, vortexed and $25 \mu \mathrm{l}$ of plasma was added directly to cortisol antibody coated tubes, followed by the addition of $500 \mu 1$ of cortisol[ $\left[^{125}\right.$ ] reagent and mixed. The mixture was incubated for 45 minutes in a water bath at $37^{\circ} \mathrm{C}$. The tubes were centrifuged and decanted and radioactivity was determined in an automatic gamma-counter (PerkinElmer, Model 1470). The minimum detection limit of this technique is $0.3 \mu \mathrm{g}$ of cortisol per $\mathrm{dL}$ of plasma. The intraassay and interassay coefficient of variance are $5.3 \%$ and $11.5 \%$ respectively.

Data Analysis: Demographic measures for individuals included in this study were developed using SAS statistical software (Cary, NC: SAS, Inc.). Growth curve models were estimated using Mplus software (Los Angeles: Muthen \& Muthen). Natural log transformed values of cortisol concentrations at the five time points were used to estimate a growth curve model that included level and shape terms. Coefficients for the shape term were empirically derived through a process of model testing after having visually inspected the actual curves of cortisol response for the groups of HIV- 1- and HIV-1+ individuals and for those with and without a history of intravenous drug use. After the growth curve model was estimated, it was further evaluated after inclusion of potentially confounding variables in the model through regressing them on the level and shape terms. This procedure allowed the model to take into account differences in cortisol level and pattern of stress response related to history of intravenous drug use, gender, age, abstinence from cocaine, recovering status and depression. Each of these variables has been shown to be related to hypothalamicpituitary-adrenal (HPA) axis response previously ${ }^{[3,18,19]}$. The direct and indirect relation of HIV serostatus on cognitive performance on the HDS was then evaluated by estimation of the direct and indirect, or mediating, relation of HIV status to HDS score. The indirect relation was evaluated via both the level and shape terms in the model (Fig. 1).

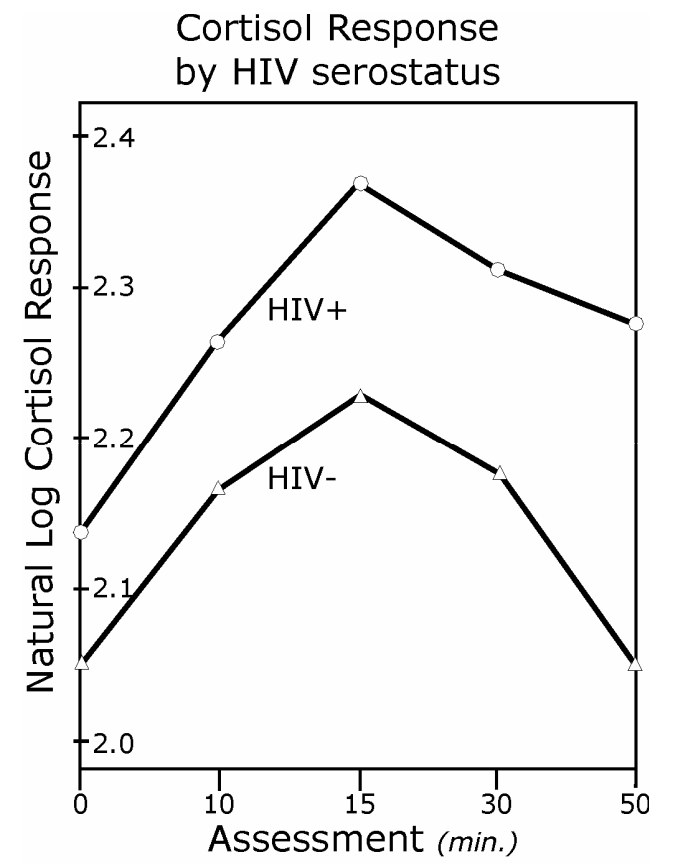

Fig. 1: Pattern of cortisol response for HIV-1 infected and uninfected individuals without correction for confounding variables

\section{RESULTS}

Descriptive data for the sample included in data analyses are presented in Table 1. Pattern of cortisol response to cold pressor challenge in individuals with and without HIV-1 infection is presented in Fig. 1. It can readily be seen that pattern of response for those with and without HIV-1 infection are substantially different. Given the number of potentially confounding variables that might have affected group differences, it was judged important to assess the effect of HIV-1 infection on cortisol function with a more complex strategy. This strategy, the latent growth curve (LGC) model, allowed simultaneous assessment of both level and pattern of cortisol response and the extent to which cortisol response was related to cognitive performance.

The LGC model that tested the hypothesis that cortisol response mediates the relationship between cognition and HIV-1 infection is graphically illustrated in Fig. 2. It can be seen that, after adjusting the HDS score for age, education, gender, intravenous drug using status and depression, the relationship between HIV status and HDS score remains significant. In addition, the relationship between cortisol response and HDS score is significant, as well as the relationship between HIV status and cortisol response. 
Table 1: Descriptive information and group comparisons

\begin{tabular}{lllll}
\hline Variable & HIV- & HIV + & $t(d f)$ & $p$ \\
\hline Age in years, mean (SD) & $33.9(8.6)$ & $39.1(5.3)$ & $-5.50(291)$ & $<0.001$ \\
Education in years, mean (SD) & $12.8(2.2)$ & $11.6(2.1)$ & $4.47(291)$ & $<0.001$ \\
BDI total, mean (SD) & $8.4(9.3)$ & $12.4(10.0)$ & $-3.40(288)$ & $<0.001$ \\
HDS total, mean (SD) & $9.4(2.5)$ & $7.1(3.1)$ & $6.64(291)$ & $<0.001$ \\
Cocaine abstinence in weeks, mean (SD) & $1,116.0(1060.3)$ & $145.9(377.0)$ & $8.60(253)$ & $<0.001$ \\
\hline Variable & HIV- & HIV+ & $\chi^{2}(d f)$ & $p$ \\
\hline Men/women & $89 / 103$ & $64 / 37$ & $7.68(1)$ & 0.01 \\
Recovering no/yes & $137 / 55$ & $101 / 0$ & $35.62(1)$ & $<0.001$ \\
IV drug use no/yes & $130 / 62$ & $0 / 101$ & $122.93(1)$ & $<0.001$ \\
White/Black/Hispanic & $43 / 92 / 57$ & $12 / 77 / 12$ & $22.01(2)$ & $<0.001$ \\
\hline
\end{tabular}

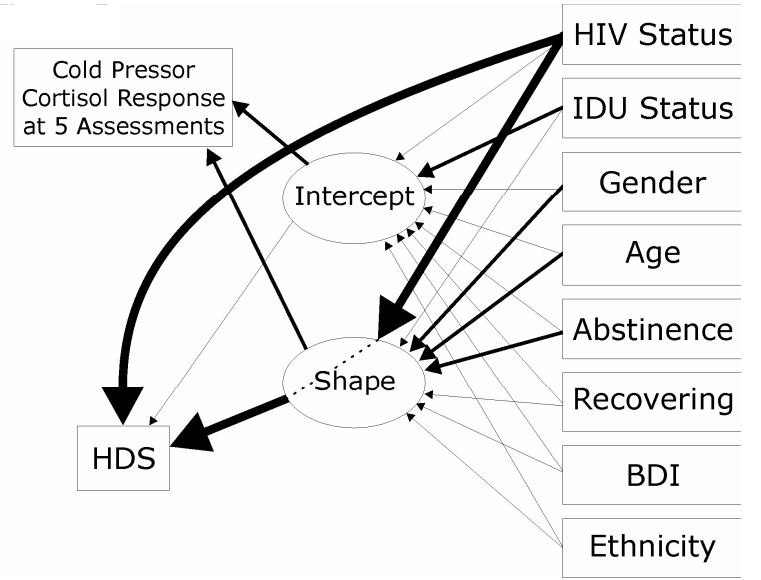

Fig. 2: Lines indicate relations estimated in the model. Bold lines are statistically significant $(p s<0.05)$. Extra bold lines between HIV status and HDS scores indicate the direct and indirect paths tested in the model. Explanation of variables: HIV Status = whether subject was HIV seropositive; IDU status $=$ whether subject had a history of injecting drug use; Gender is self explanatory; Age = age in years; Abstinence = time since last use of cocaine; Recovering = whether IDUs described themselves as in recovery; $\mathrm{BDI}=$ total score on the Beck Depression Inventory

\section{DISCUSSION}

The purpose of this study was to test the hypothesis that the cortisol response to stress mediates the relation between HIV serostatus and cognitive function. Our results show that differences in the pattern of cortisol response to stress are related to HIV serostatus and that the relation between serostatus and HDS performance may itself be partially mediated by the indirect relation between the serostatus and cognitive performance by way of cortisol response. These results are thus consistent with our hypothesis and imply that deficits in cognitive function related to HIV-1 infection may result from neuroendocrine system dysfunction.

Exposure to elevated cortisol levels is associated in healthy adults with physical and psychological distress and can cause reversible impairments in some aspects of verbal memory performance ${ }^{[20]}$. Our findings show similar results since the mHDS also measures declarative verbal memory. Glucocorticoid (GC) levels (reflected by cortisol levels in humans) are elevated for prolonged periods in HIV-1 infection ${ }^{[21]}$. Given the close significant relation between cortisol response to stress, HIV serostatus and cognitive function, the findings from the present study present suggestive parallels to these previous findings. It thus seems reasonable to infer that dysregulated cortisol response in HIV infection may be an important link between widely-observed cognitive deficits to infection.

Although the exact mechanism for the relations among serostatus, cortisol response and HDS is unclear, high GC levels are known to have adverse effects on the $\mathrm{CNS}^{[7]}$. GC-mediated effects are pronounced on several areas of the brain, especially the hippocampus, leading to atrophy of hippocampal dendrites and neuron loss. Studies using high resolution MRI show that decreases in hippocampal volumes in depression, Cushing $=\mathrm{s}$ syndrome and PTSD (all conditions in which individuals are known to have high cortisol levels) are related to the duration of disease ${ }^{[22]}$. Although such high resolution studies have not yet been extensively carried out in HIV-1+ individuals, investigations using MRI even at low-resolution show widespread neuroanatomic defects among individuals with $\mathrm{MCMD}^{[23,24]}$. Multiple lines of evidence thus indicate that chronic exposure to high cortisol levels can lead to neuroanatomic deficits, the behavioral effects of which are revealed in psychometric testing.

$\mathrm{GC}$, the end-hormone of the HPA axis, (cortisol in humans and corticosterone in rats), is secreted in an ultradian pulsatile fashion with secretory episodes occurring with a constant frequency but with a variable amplitude. Increased level of GC depend on the amplitude of these episodes as pulsatile frequency remains constant. The circadian, as well as stressrelated increases in GC levels, result from increases in the amplitude of secretory episodes ${ }^{[25]}$. These activities are regulated at the level of the hypothalamus, in particular in the parvocellular component of the paraventricular (PVN) nuclei. In normals the neuroendocrine stimulation of increased GC levels is 
counteracted by corticosteroid receptor mediated negative feedback which promptly brings down the GC levels. Data suggest that three separate regulatory mechanisms coordinate the adrenal secretions. The first mechanism is the circadian rhythm of basal activity and is derived from the suprachiasmatic nucleus. The second mechanism deals with stress induced changes in the HPA axis and involve afferent inputs from brain regions including noradrenergic enervation from brain stem A1 and A2 cell groups and the locus ceruleus, amygdala, cerebral cortex and hippocampus. The third mechanism for the negative feedback is exerted through corticosteroid receptors of both high (type I, mineralocorticoid receptors, $\mathrm{MR}, \mathrm{k}_{\mathrm{D}}=0.5 \mathrm{nM}$ ) and low affinities (type II, glucocorticoid receptors, GR, $\mathrm{k}_{\mathrm{D}}=$ $5.0 \mathrm{nM}$ ). These systems are necessary to regulate a wide range of GC levels. Recent studies show that corticosteroid receptors are present on lymphocytes and their mRNA gene expression can be used to quantify their activity. However, little data are available on corticosteroid-receptors in HIV-1 infection. Our unpublished data show that HIV-1 infection may be associated with decreased corticosteroid receptor mRNA expression in HIV-1 infection.

Under conditions of prolonged cortisol secretion, HPA axis failure can take place either due to glial hypertrophy or to a depletion of adrenergic stores in the locus ceruleus neurons and a possible GC-induced neuronal loss in the hippocampus ${ }^{[18,26]}$. Several reports suggest that the effects of cortisol on cognition can be paradoxical since both the high (as in Cushing's syndrome) and low levels of cortisol (Addison's disease) have been reported in subjects with cognitive dysfunctions ${ }^{[27,28]}$. Emerging evidence suggests that there may also be a disturbance in the circadian rhythm of GC in HIV-1 infection that leads to higher plasma cortisol levels in the evening ${ }^{[29,30]}$. GC resistance is reported to exist in about $17 \%$ of HIV-1+ subjects and may result in a decoupling of circulating cortisol levels and corticosteroid receptor activity ${ }^{[31]}$. GC resistance can result from: a) a defect in translocation of GR receptors from the cytoplasm to the nucleus ${ }^{[32,33]}$ b) an increased and prolonged $\mathrm{CRH}$ concentration presumably due to defective glucocorticoid negative feedback; and c) inflammatory cytokine induced hypercortisolemia. In addition, a novel complication has been introduced in this area by studies showing that an HIV auxiliary protein, $v p r$ (viral protein R), interacts with glucocorticoid type II receptors ${ }^{[34,35]}$. Recent in vitro transfection studies ${ }^{[36]}$ looking at HIV-vpr gene expression suggest that $v p r$ can induce cortisol resistance.

These lines of research show that a number of possible mechanisms could account for the observed mediation of cognitive status and HIV-1 infection via cortisol response. Chronically high GC levels may lead to neurohormonal dysregulation by way of several pathways. The same chronically high GC levels may also be related to neuroanatomic changes in brain areas related to cognitive function. These relations may thus account for the links shown in this study HIV-1 between serostatus, cortisol response and cognitive function. Although additional investigations on the role of the HPA axis in the development of cognitive dysfunction in HIV-1 infection will be required to more conclusively establish this link, this study shows that cortisol response and cognitive function may be closely related in individuals with HIV-1 infection.

\section{ACKNOWLEDGEMENTS}

This study was supported by grant R01 DA12792 and DA13550 to Dr. Mahendra Kumar.

\section{REFERENCES}

1. Kumar, M., A.M. Kumar, D. Waldrop, M.H. Antoni and C. Eisdorfer, 2003. HIV-1 infection and its impact on the HPA axis, cytokines and cognition. Stress, 6: 167-72.

2. Jin, W.D., A.L. Boutillier, M.J. Glucksman, S.R. Salton, J.P. Loeffler and J.L. Roberts, 1994. Characterization of a corticotropin-releasing hormone-responsive element in the rat proopiomelanocortin gene promoter and molecular cloning of its binding protein. Mol. Endocrinol., 8: 1377-88.

3. Licinio, J., P.W. Gold and M.L. Wong, 1995. A molecular mechanism for stress-induced alterations in susceptibility to disease. Lancet, 346: 104-6.

4. Gold, P.W., personal communication.

5. Gurevitch, D., B. Siegel, M.S. Dumlao, E. Perl, P. Chaitin, C.A. Bagne and G.F. Oxenkrug, 1989. The relationship between cognitive impairment plasma cortisol levels and HPA responsibility to dexamethasone in dementia. Prog. Clin. Biol. Res., 317: 175-87.

6. McEwen, B.S. and R.M. Sapolsky, 1995. Stress and cognitive function. Curr. Opin. Neurobiol., 5: 205-16.

7. Sapolsky, R.M., 1996. Why stress is bad for your brain. Science, 273: 749-50.

8. Glasgow, B.J., K.D. Steinsapir, K. Anders and L.J. Layfield, 1985. Adrenal pathology in the acquired immune deficiency syndrome. Am. J. Clin. Pathol., 84: 594-7.

9. Kumar, M., A.M. Kumar, R. Morgan, J. Szapocznik and C. Eisdorfer, 1993. Abnormal pituitary-adrenocortical response in early HIV-1 infection. J. Acquir. Immune. Defic. Syndr., 6: 615.

10. Membreno, L., I. Irony, W. Dere, R. Klein, E.G. Biglieri and E. Cobb, 1987. Adrenocortical function in acquired immunodeficiency syndrome. J. Clin. Endocrinol. Metab., 65: 482-7. 
11. Merenich, J.A., M.T. McDermott, A.A. Asp, S.M. Harrison and G.S. Kidd, 1990. Evidence of endocrine involvement early in the course of human immunodeficiency virus infection. J. Clin. Endocrinol. Metab., 70: 566-71.

12. Villette, J.M., P. Bourin, C. Doinel, I. Mansour, J. Fiet, P. Boudou, C. Dreux, R. Roue, M. Debord and F. Levi, 1990. Circadian variations in plasma levels of hypophyseal, adrenocortical and testicular hormones in men infected with human immunodeficiency virus. J. Clin. Endocrinol. Metab., 70: 572-7.

13. Kumar, M., R. Morgan, J. Szapocznik and C. Eisdorfer, 1991. Norepinephrine response in early HIV infection. J. Acquir. Immune. Defic. Syndr., 4: 782-6.

14. Malarkey, W.B., I.M. Lipkus and J.T. Cacioppo, 1995. The dissociation of catecholamine and hypothalamic-pituitary-adrenal responses to daily stressors using dexamethasone. J. Clin. Endocrinol. Metab., 80: 2458-63.

15. Duncan, T.E., S.C. Duncan, L.A. Strycker, F. Li and A. Alpert, 1999. Introduction of latent variable growth curve modeling. Lawrence Erlbaum.

16. First, M.B., R.L. Spitzer, M. Gibbon and J.B.W. Willams, 1995. Structured Clinical Interview for DSM-IV Axis I Disorders - Patient Edition (SCIDI/P Version 2.0). New York State Psychiatric Institute, Biometrics Research Department.

17. Skolasky, R.L., D.R. Esposito, O.A. Selnes, C. Power and J. McArthur, 1998. Modified HIV dementia scale: Accurate staging of HIVassociated dementia. J. NeuroVirol., 4: 366.

18. Galina, Z.H. and Z. Amit, 1986. Stress-induced analgesia: its effects on performance in learning paradigms. Ann. N.Y. Acad. Sci., 467: 238-48.

19. Lupien, S.J., M. de Leon, S. de Santi, A. Convit, C. Tarshish, N.P. Nair, M. Thakur, B.S. McEwen, R.L. Hauger and M.J. Meaney, 1998. Cortisol levels during human aging predict hippocampal atrophy and memory deficits. Nat Neurosci., 1: 6973.

20. Newcomer, J.W., G. Selke, A.K. Melson, T. Hershey, S. Craft, K. Richards and A.L. Alderson, 1999. Decreased memory performance in healthy humans induced by stress-level cortisol treatment. Arch. Gen. Psychiat., 56: 527-33.

21. Mayo, J., J. Collazos, E. Martinez and S. Ibarra, 2002. Adrenal function in the human immunodeficiency virus-infected patient. Arch. Intern. Med., 162: 1095-8.

22. Sheline, Y.I., P.W. Wang, M.H. Gado, J.G. Csernansky and M.W. Vannier, 1996. Hippocampal atrophy in recurrent major depression. Proc. Natl. Acad. Sci. USA, 93: 3908-13.
23. Hall, M., R. Whaley, K. Robertson, S. Hamby, J. Wilkins and C. Hall, 1996. The correlation between neuropsychological and neuroanatomic changes over time in asymptomatic and symptomatic HIV-1-infected individuals. Neurology, 46: 1697-702.

24. Kieburtz, K., L. Ketonen, C. Cox, H. Grossman, R. Holloway, H. Booth, C. Hickey, A. Feigin and E.D. Caine, 1996. Cognitive performance and regional brain volume in human immunodeficiency virus type 1 infection. Arch. Neurol., 53: 155-8.

25. Chrousos, G.P., 2000. The stress response and immune function: clinical implications. The 1999 Novera H. Spector Lecture. Ann. N.Y. Acad. Sci., 917: 38-67.

26. Munck, A., P.M. Guyre and N.J. Holbrook, 1984. Physiological functions of glucocorticoids in stress and their relation to pharmacological actions. Endocr. Rev., 5: 25-44.

27. McEwen, B.S., 1987. Glucocorticoid-biogenic amine interactions in relation to mood and behavior. Biochem. Pharmacol., 36: 1755-63.

28. McEwen, B.S., 1992. Steroid hormones: effect on brain development and function. Horm. Res., 37 (Suppl. 3): 1-10.

29. Malone, J.L., E.C.3rd Oldfield, K.F. Wagner, T.E. Simms, R. Daly, J. O'Brian and D.S. Burke, 1992. Abnormalities of morning serum cortisol levels and circadian rhythms of CD4+ lymphocyte counts in human immunodeficiency virus type 1-infected adult patients. J. Infect. Dis., 165: 185-6.

30. Kumar, M., Unpublished Data.

31. Norbiato, G., M. Bevilacqua, T. Vago and $M$. Clerici, 1996. Glucocorticoids and interferon-alpha in the acquired immunodeficiency syndrome. J. Clin. Endocrinol. Metab., 81: 2601-6.

32. Miller, A.D., 1996. Cell-surface receptors for retroviruses and implications for gene transfer. Proc. Natl. Acad. Sci. USA, 93: 11407-13.

33. Norbiato, G., M. Bevilacqua, T. Vago, G. Baldi, E. Chebat, P. Bertora, M. Moroni, M. Galli and N. Oldenburg, 1992. Cortisol resistance in acquired immunodeficiency syndrome. J. Clin. Endocrinol. Metab., 74: 608-13.

34. Kino, T., A. Gragerov, J.B. Kopp, R.H. Stauber, G.N. Pavlakis and G.P. Chrousos, 1999. The HIV1 virion-associated protein vpr is a coactivator of the human glucocorticoid receptor. J. Exp. Med., 189: 51-62.

35. Refaeli, Y., D.N. Levy and D.B. Weiner, 1995. The glucocorticoid receptor type II complex is a target of the HIV-1 vpr gene product. Proc. Natl. Acad. Sci. USA, 92: 3621-5.

36. Chrousos, G.P. and T. Kino, 1997. The HIV VPR gene product can cause glucocorticoid hypersensitivity or resistance: implications for the pathogenesis and clinical presentation of AIDS. Psychoneuroendocrinology, 22: s160. 